\title{
A solar flare scenario based on multi-wavelength observations
}

\author{
W. Q. Gan, Y. P. Li, H. Li, X. F. Yu, and Q. S. Du \\ Purple Mountain Observatory, Chinese Academy of Sciences, Nanjing 210008, China \\ email: wqgan@pmo.ac.cn
}

The 1N/M7.6 flare of 24 October 2003 at S19E72 were well observed by RHESSI, TRACE, NORH, and the Spectrograph at the Purple Mountain Observatory. After analyzing all the data collected, we established a scenario of the flare and compared it with the standard cartoon of solar flares (e.g., Dennis 1988).

The GOES records the start, maximum, and end time of the flare as 0227 UT, 0254 UT, and 0314 UT. But both the lightcurves of RHESSI and GOES exhibit two bursts during the flare (Figure 1). The second burst is more energetic and overlaps the first burst just after its maximum. The morphologies at the lower atmosphere are addressed by the bright ribbons of TRACE $1600 \AA$, TRACE $195 \AA$, and $\mathrm{H} \alpha$, as well as the hard X-ray footpoints, while the information on the higher atmosphere by TRACE $195 \AA$ and NORH $34 \mathrm{GHz}$ images, and the soft X-ray sources. The scenario of the flare can be described as follows:

For the first burst, the loop-arch in TRACE $195 \AA$ (magnetic structure) has already been there before the flare onset (left panel of Figure 2). When the flare happens, the soft $\mathrm{X}$-ray bright patch appears at the top of the original loop-arch, while the original looparch disappears due to a higher temperature (middle panel of Figure 2). Meanwhile the response in the lower atmosphere is manifested by $\mathrm{H} \alpha$ and TRACE $1600 \AA$ brightening which is spatially coincident with the foot-ribbon of the original loop-arch. During the impulsive and maximum phase, a hard X-ray source appears in the lower atmosphere only for a short period of about $30 \mathrm{~s}$, and soon afterwards the $31.6-42.2 \mathrm{keV}$ hard X-ray source moves to the upper part. The TRACE $195 \AA$ loop-like structure (not the original loop-arch) brightens and seems to be a little beneath the soft X-ray source.

For the second burst, during the impulsive and maximum phase, one hard X-ray source appears first in one point (left panel of Figure 3) where TRACE 195 $A$, TRACE $1600 \AA$, and $\mathrm{H} \alpha$ brighten simultaneously. This hard X-ray source moves southward with time and TRACE $195 / 1600 \AA$ bright points move correspondingly. Then the hard X-ray source becomes two footpoints (middle panel of Figure 3) which are located at the two ribbons of TRACE $1600 \AA$ and $\mathrm{H} \alpha$. There is an obvious time delay in soft X-ray morphology with respect to the appearance of hard X-ray source, and the soft X-ray source is more extended and higher in altitude than that of the first burst (right panel of Figure 3). During the decay phase the soft X-ray source moves upward gradually, while the heated lower atmosphere manifested by TRACE $1600 \AA$ and $\mathrm{H} \alpha$ ribbons sustains for quite a long time. A post-flare loop-arch appears long time later, which looks similar to the original loop-arch before the first burst (right panel of Figure 2).

These patterns are generally consistent with the standard cartoon of solar flares, as studied by Gan et al. (2003) and Li \& Gan (2004) for other two flares. Especially, the first burst tends to be of thermal origin, while the second burst of non-thermal origin. The result also suggests that the original loop-arch is the basic magnetic structure where the reconnection happens. 

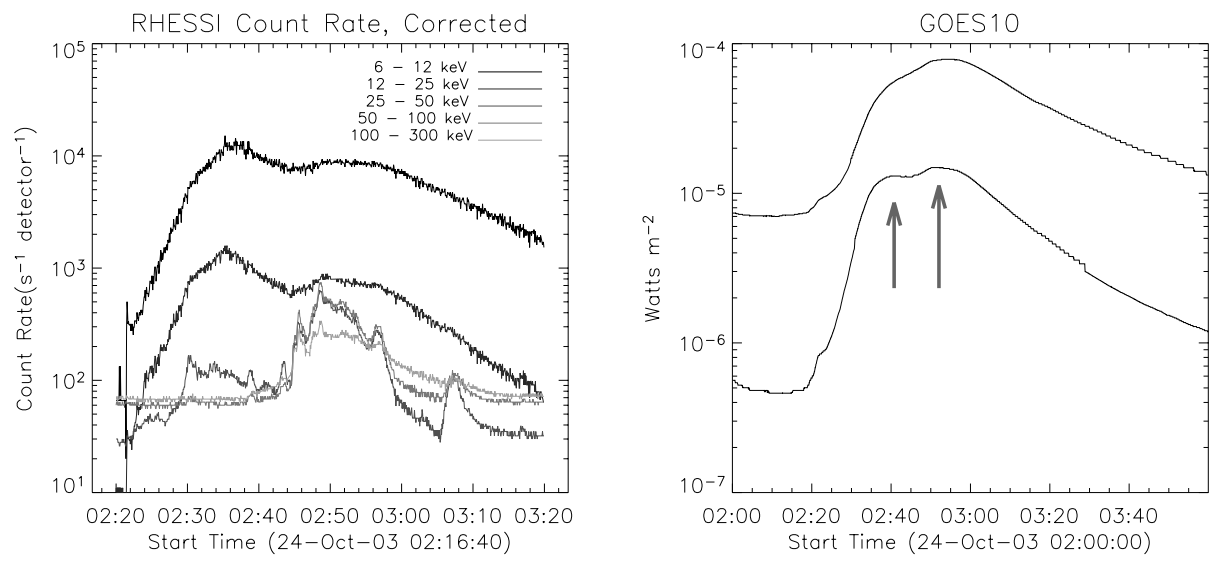

Figure 1. Lightcurves of RHESSI and GOES for the 1N/M7.6 flare. The arrows indicate two bursts.
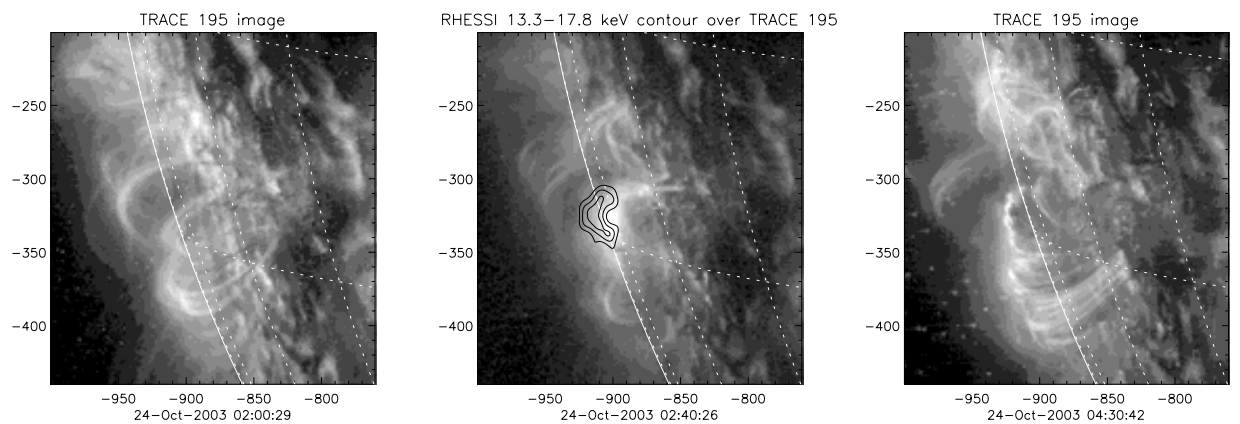

Figure 2. TRACE 195 morphology before (left) and after (right) the flare. Middle panel shows the soft X-ray source (contour) overlain on TRACE 195 for the peak time of burst 1.
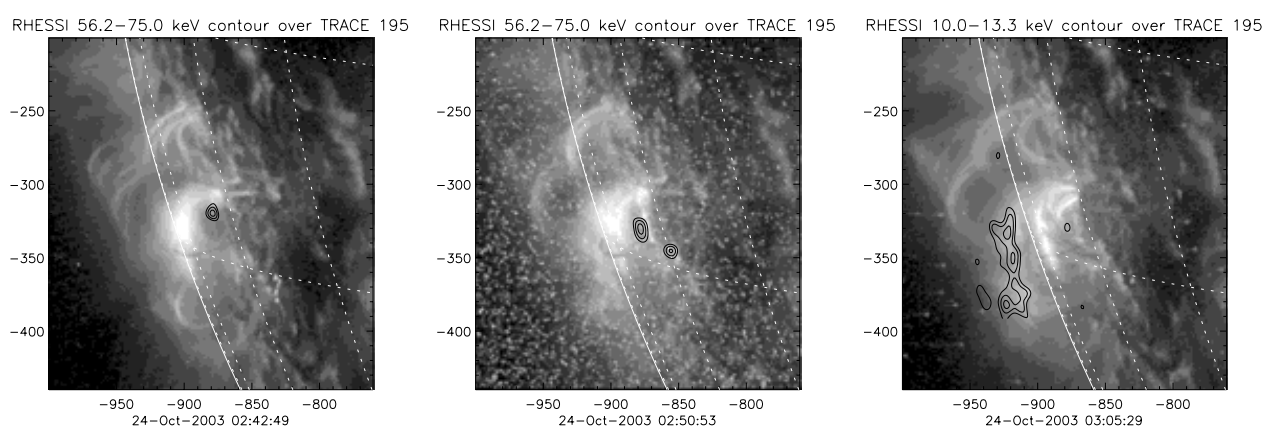

Figure 3. Some evolutionary episodes of burst 2

\section{References}

Dennis, B. R. 1988 Solar Phys. 118, 49-94.

Gan, W. Q. et al. 2003 Adv. Space Res. 32, No.12, 2471-2476.

Li, Y. P.\& Gan, W. Q. 2004 Chin. Astron. Astrophys. 28, 409-416. 